\title{
PID CONTROLLER DESIGN FOR A ONE-DIMENSIONAL HEATED ROD USING ORTHOGONAL COLLOCATION
}

\author{
A. O. F. Williams ${ }^{1, *}$ and V. O. Adeniyi ${ }^{2}$

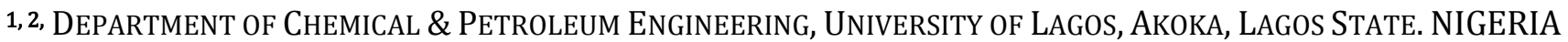 \\ E-mail addresses: ${ }^{1}$ afwilliams@unilag.edu.ng, ${ }^{2}$ vadeniyi@unilag.edu.ng
}

\begin{abstract}
This paper presents the design of PID-type controllers for a one-dimensional, distributed heat conduction system starting from the parabolic partial differential equation modelling the system. The partial differential equation modelling the system was lumped using the orthogonal collocation method resulting in third-order lumped model. This lumped model was then used for the controller design based on a method of PID controller design previously developed for higher-order systems by the authors. The results of closed-loop simulations demonstrate the superior performance of the PID-type controllers so designed. The approach presented is quite general and may be used to carry out PID-type controller designs for other single-input, single-output distributed parameter systems.
\end{abstract}

Keywords: Distributed parameter system, heat conduction, partial differential equation, early lumping, orthogonal collocation, PID controller design, Closed loop simulation.

\section{INTRODUCTION}

The dynamics (or transient response behaviour) of heat transfer and other transport processes are modeled by partial differential equations (based on first principles or mechanistic modeling approach), and therefore belong to the class of systems known as distributed parameter systems (DPS). In these systems, the state/output and/or the input may vary both spatially and temporarily $[1,2]$.

Some practical examples of heat-transfer processes may not necessarily be one-dimensional. However, when the diameter (or width) to length ratio is small (and this the case for many fins and rod applications), it is found that the assumption of the system being onedimensional is a reasonable one which simplifies the analysis of the problem without significant loss in accuracy. Common examples of this include: heat transfer in fins, rods and other extended surfaces which are often utilized to increase the heat-transfer rate [2 4].

There are two major approaches for the design of feedback controllers for distributed parameter systems, namely: early and late lumping approaches [1]. Like many other processes that are modeled by partial differential equations i.e. distributed parameter systems (DPS), the control design problem for many dynamic heat transfer processes can effectively be reduced to the design of PID-type controllers based on the "early lumping" approach. The alternative approach based on "late lumping" involves the application of distributed parameter system theory and the mathematical rigour required by this approach is not attractive for industrial deployment for many practical processes $[1,5]$. The attraction of the "early lumping" approach is the use of a simpler model that closely approximates the original DPS, and consequently leads to less number of equations to be solved. Additionally, one can then employ any of the large body of controller design methods for lumped parameter (LP) systems with much ease.

Although a classical method such as the finite difference technique can be readily applied to lump a DPS in the spatial variable(s), it is well known to be computationally inefficient (and often results in a lumped parameter system of high order) compared with other techniques such as the method of weighted residuals, of which the orthogonal collocation method is a popular one[6,7]. Other approaches to lumping a DPS are the use of the modal decomposition method [1]; use of Laplace transform method to derive transfer functions of the system which are irrational functions, and then approximating the irrational transfer functions by a rational one in order to carry out controller design $[8,9]$. A brief review of the various 
modeling and lumping techniques for DPS is presented in [10].

In this paper, the objective is to explore the use of the method of orthogonal collocation to lump the dynamic model of a one-dimensional heated rod, and to subsequently use a modified Internal Model Control (IMC) approach to design PID-type controllers for the point temperature control at the designated position along the heated rod. The approach adopted allows to easily compute a low-order lumped model which is then directly employed in the controller design using the modified IMC-PID method previously developed by the authors. Although the modal decomposition method could be applied to the system studied here, it is to be noted that the level of mathematics involved is often a discouraging factor for industrial practitioners. Moreover, the method is only applicable to linear second-order partial differential equations which have real and discrete spectrum of modes (eigenvalues), and with a spatial operator that can be made self-adjoint[1]. Thus, the motivation for the use of the orthogonal collocation method to lump the system studied here is that it is quite general, and together with the PIDdesign method employed, the entire process is much easily programmed for automatic computation on a computer, thereby making it much more attractive for the practitioners in industry.

Although several advanced design techniques such as internal model control (IMC) and model predictive control (MPC) are available, the bulk of process control applications are still largely carried out using PID-type controllers. Consequently, the design of SISO controllers, especially of the PID-type, for SISO DPS systems is of practical importance, and continues to attract the attention of practitioners and researchers alike. As pointed out by [11], the popularity and wide acceptability of PID controllers in many process control applications is so because of its structural simplicity, constant disturbance cancellation, error-free tracking of constant setpoints, and reduced sensitivity to parameter variations. A similar view was also expressed by [12] who note that "the wide diffusion of conventional controllers (PI-PID) in process control
( $\geq 95 \%$ ccording to [13]) cannot be explained only in terms of resistance to change from operators or lack of challenging problems to face. The reasons for that have to be found in the major simplicity, reliability and favorable ratio between performance and cost." These facts explain why methods for the design/tuning of PID-type controllers abound in the literature.

The classical methods for the design of PID controllers are the semi-empirical rules $[14,15]$. These methods give controller settings which are often found to be more under-damped than desired in many applications [16]. As a result, extensive efforts have to be used (in a trial-and-error fashion) to fine-tune the controller online in order to obtain acceptable closed-loop response behavior of the controlled process. Thus methods for the systematic design of PID controllers with better performance continue to be active areas of research $[12,17-29]$. A brief survey of some of the recent research trends for PID controllers can be found in [30], while [31-34] are some of the papers in the literature on the design or application of PID-type controllers to distributed parameter systems.

\section{METHODOLOGY}

\subsection{The One-Dimensional Heated Rod and its Mathematical Model}

Consider the schematic diagram in Figure 1 in which a thin metal rod has one end in a water bath maintained at $T_{0} .{ }^{0} \mathrm{C}$, and the other end inserted into a steam chest. Air at $T_{0} .{ }^{0} \mathrm{C}$, is blowing transversely across the rod. The temperature of the right-hand end is assumed fixed at $T_{0} \cdot{ }^{0} C$, while the temperature of the left-hand end may be controlled by adjusting the steam pressure [1].

Making the following assumptions: (i) that density, $\rho$, specific heat, $C_{p}$, the thermal conductivity, $k=k_{0}$, of the system are constant, (ii) that the metal rod has a uniform cross-sectional area, $A$, (iii) the diameter of the rod is small compared to its length, then by writing a microscopic energy balance on the system, it is straight forward to show that the mathematical model of the system can be put in the dimensionless form [1]

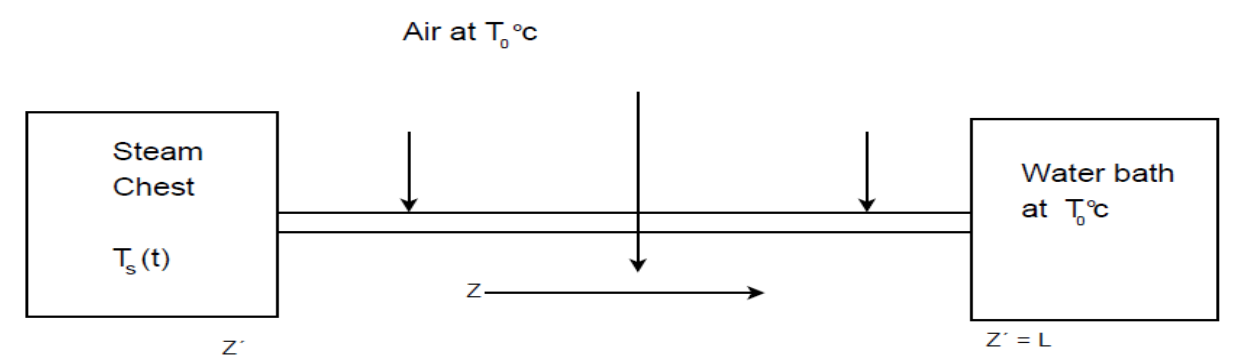

Figure 1: A thin metal rod being heated at one end and the other end maintained at a constant temperature [1] 


$$
\frac{\partial y}{\partial t}=\frac{\partial^{2} y}{\partial t^{2}}-\beta_{0} y
$$

subject to the following initial and boundary conditions:

$$
\begin{gathered}
\text { At } \quad t=0, \quad y(z, 0)=\bar{y}(z) \\
\text { At } \quad z=0, \quad y(0, t)=u \quad z=1, \quad y(1, t)=0
\end{gathered}
$$

where the following dimensionless/deviation variables are defined as:

$$
\begin{gathered}
y=\frac{T-T_{0}}{T_{0}}, \quad u(t)=\frac{T_{s}-T_{0}}{T_{0}}, \\
\bar{y}(z)=\frac{\bar{T}(z)-T_{0}}{T_{0}}, \quad \beta_{0}=\frac{h^{\prime} L^{2}}{k_{0}} \\
z=z^{\prime} / L, \quad t=\frac{t^{\prime} K_{0}}{\rho C_{P} L^{2}}
\end{gathered}
$$

Here, $T_{s}\left(t^{\prime}\right)$ is the adjustable temperature of the steam chest, $h^{\prime}=h S / A, h$ is the heat transfer film coefficient between the metal surface and the surrounding air and $S$ is the surface area for heat transfer per unit length of the metal rod.

\subsection{Lumped Model of the One-Dimensional Heated Rod Using Orthogonal Collocation}

To covert the PDE model, Eq. (1) of the heat conduction system into a lumped parameter (LP) model, using orthogonal collocation, a trial solution of the following form is assumed [35]:

$$
y(z, t)=\sum_{k=0}^{n+1} \ell_{k}(z) y_{k}(t)
$$

Substituting the trial solution into Eq. (1) and setting the residuals to zeros at the collocation points, leads to:

$$
\frac{d y_{j}}{d t}=\sum_{k=0}^{n+1} B_{j k} y_{k}-\beta_{0} y_{j}, j=1,2, n
$$

in which $A_{j k}$ and $B_{j k}$ are the first and second collocation matrices given by [35]

$$
\begin{aligned}
& A_{j k}=\left.\frac{d \ell_{k}(z)}{d z}\right|_{z=z_{j}} \\
& B_{j k}=\left.\frac{d \ell_{k}(z)}{d z^{2}}\right|_{z=z_{j}}
\end{aligned}
$$

and the collocation points $z_{j}, j=0,1, K, \quad n-1$ are the roots of the orthogonal Jacobi Polynomial [35]:

$$
\begin{gathered}
\int_{0}^{1} z^{\beta}(1-z)^{a} z^{j} P_{n}^{(a, \beta)}(z) d z=0 \\
a, \beta>-1, j=0,1, n-1
\end{gathered}
$$

Using the two boundary conditions given by Eq. (3) i.e.

$$
y(0, t)=y_{0}(t)=u(t), \quad y(1, t)=y_{n+1}(t)=0
$$

$$
\frac{d y_{j}}{d t}=\sum_{k=1}^{n}\left(B_{j k}-\beta_{0} \delta_{j k}\right) y_{k}+B_{j} u(t) j=1,2, n
$$

where

$$
\delta_{j k}= \begin{cases}1 & j=k \\ 0 & j \neq k\end{cases}
$$

Eq. (12) can be put in the standard state-space form:

$$
\begin{gathered}
\dot{x}=A_{1} \mathrm{x}+b_{1} \mathrm{u} \\
y=c \mathrm{x}
\end{gathered}
$$

where the system states, $\mathrm{x}$, and the matrices $\mathrm{A}_{1}, b_{1}$ and $C$ (for measurement at the point along the length where temperature is to be controlled) are readily determined.

Note that compared with the modal decomposition and the Laplace transform approaches, the attraction of the orthogonal collocation lumping approach is that there are high quality computer routines [35] that allows the generation of the required collocation points and matrices automatically once the input parameters ( $\alpha, \beta$ and the number of collocation points $(n))$ are specified. Thus, the entire lumping step, together with the PID controller design method to be shortly presented, can be readily embedded into a computeraided design environment which can then be easily used by industrial practitioners. Efforts are currently on going to develope such a computer-aided design environment for distributed parameter systems, and this shall be the subject of a future paper.

\subsection{PID Control Design/Tuning Method}

Details of the PID-type controller design/tuning method employed are available in [20]. Therefore, only an overview is presented here. Let the model of the process for which a PID controller is to be designed, be represented as

$$
\tilde{g}(s)=\frac{\sum_{i=0}^{\grave{M}} \grave{a}_{i} s^{i}}{\sum_{i=0}^{N} b_{i} s^{i}} \quad \widehat{M}<N
$$

in which it has been assumed that any pure time delays originally present in the system model has been rationalized using, for example, a first-order Padé polynomial.

By using the IMC design method[17, 18, 36] to design the IMC controller $g_{I}$ for Eq. (16), and then transforming this into the classical controller $g_{c}$ (through the equivalence: $g_{c}=g_{r}\left(1-\tilde{g} g_{1}\right)$ ), it can be shown that the resulting controller transfer function for $g_{c}$ can be simplified to the form: 


$$
g_{c}=\frac{\sum_{i=0}^{N} b_{i} s^{i}}{\sum_{i=1}^{N} a_{i} s^{i}}
$$

in which the numerator coefficients, $b_{i}$ are those of the denominator of the original model, Eq. (17); while the denominator coefficients, $a_{i}$, now depend on some of the coefficients resulting from the factorization of $\tilde{g}(s)$. into $\tilde{g}_{+}(s)$ and $\tilde{g}_{-}(s)$, and the IMC filter parameter, $\varepsilon$ [17].

The controller, $g_{c}$, given by Eq. (17) can be very different from the classical PI/PID controllers. In the new PID-type controller design method, Eq. (17) is directly reduced to a PI or PID controller through the low- and high-frequency approximation of the controller transfer function, $g_{c}$. Depending on whether a PI, an ideal PID, or a PID controller cascaded with a first-order lag (i.e. a "real " or "practical" PID controller) is considered, different expressions for the controller parameters can be derived as shown in the following.

\subsubsection{Reduction to a PI Controller}

At low- and high-frequencies, Eq. (17) can be approximated, respectively by PI controllers, as follows:

$g_{c_{\text {low }}}=\frac{b_{1} s+b_{0}}{a_{1} S}, \quad g_{c_{\text {high }}}=\frac{b_{N} S^{N}+b_{N-1} S^{N-1}}{a_{N} S^{N}}$

Let the fraction contributed by the low-frequency approximation to the PI controller be $\varphi$, so that $(1-\varphi)$ is the fraction contributed by the highfrequency approximation, then the resulting PI controller transfer function is

$$
\begin{gathered}
g_{c_{(P I)}}=K_{c}\left(1+\frac{1}{\tau_{1}} \frac{1}{s}\right) \\
K_{c}=\frac{b_{1}}{a_{1}} \varphi+(1-\varphi) \frac{b_{N}}{a_{N}}, \\
\tau_{I}=\frac{a_{N} b_{1} \varphi+(1-\varphi) a_{1} b_{N}}{a_{N} b_{0} \varphi+(1-\varphi) a_{1} b_{N-1}}
\end{gathered}
$$

\subsubsection{Reduction to Ideal PID Controllers}

Through a similar procedure as above, the ideal PID controller approximation of Eq. (17) is:

$$
\begin{gathered}
g_{c_{(P I D)}}=K_{c}\left(1+\frac{1}{\tau_{1}} \frac{1}{S}+\tau_{D} S\right) \\
K_{c}=\frac{b_{1}}{a_{1}} \varphi+(1-\varphi) \frac{b_{N}}{a_{N}} \\
\tau_{I}=\frac{a_{N} b_{1} \varphi+(1-\varphi) a_{1} b_{N}}{a_{N} b_{0} \varphi+(1-\varphi) a_{1} b_{N-1}}
\end{gathered}
$$

$$
\tau_{D}=\frac{b_{2} a_{N}}{a_{N} b_{1} \varphi+(1-\varphi) a_{1} b_{N}}
$$

Expressions for the parameters of practical PID controllers (i.e. an ideal PID controller cascaded with a first-order filter) have also been derived from the lowand high-frequencies approximations of Eq. (17) Further details are available in [20].

\subsubsection{Controller Tuning}

For a given system, $\tilde{g}(s)$, the PI and PID controller parameters given above depend on both the filter parameter, $\varepsilon$ (which is implicit in $a_{i}$ 's) and the parameter, $\varphi$ which determines the fraction of the low- and high-frequency approximations of Eq. (17) included in the PI and PID controllers. Thus apart from $\varepsilon$, the parameter $\varphi(0 \leq \varphi \leq 1)$ provides an additional way of influencing the closed-loop response provided by the PI and PID controllers.

Now, it is obvious that the approximation of the full controller, $g_{c}$, given by Eq. (17), by a PI or PID-type controller will result in performance degradation from that attainable with Eq. (17), and may even lead to closed-loop stability problems when implemented on the original higher-order system. Thus to ensure closed-loop stability and acceptable performance, an appropriate value of the filter parameter, $\varepsilon$ must be chosen. The maximum closed-loop amplitude ratio criterion was used to ensure this, as follows: for a given value of $\varphi$, choose $\varepsilon^{\text {å }}$ such that:

$F=\max _{s=j \omega}\left|\frac{g^{\%}(s) g_{c}\left(s, \varepsilon^{*}, \varphi\right)}{1+g^{\%}(s) \quad g_{c}\left(s, \varepsilon^{*}, \varphi\right)}\right|-M^{*}=0$

where $M^{*}$ is the desired maximum closed-loop amplitude ratio. A simple algorithm to solve this problem is the Interval Bisection Method for which a FORTRAN function subprogram ZEROIN is presented in[37]. The problem could also be solved in the MATLAB, SCILAB and MATHEMATICA environments.

\subsection{Application to the One-Dimensional Heat Conduction System}

\subsubsection{Lumped Model and Transfer Function}

Based on the recommendations of [35] for parabolic partial differential equations, $a=0, \beta=0$ were chosen to generate the collocation points and the collocation matrices required for lumping the system. Although not presented here due to page size limit considerations, the dynamic response of the resulting lumped system was explored for various number of collocation points from 2 to 5 , and compared these with the analytical solution obtained by the modal 
decomposition technique which can easily be shown to be given by:

$$
\begin{gathered}
y(z, t)=\frac{\bar{u}_{2} \sinh \sqrt{\beta_{0}}(1-z)}{\sqrt{\beta_{0}}} \\
+\sum_{k=1}^{\infty} \frac{2 k \pi\left(\bar{u}_{1}-\bar{u}_{2}\right)}{k^{2} \pi^{2}+\beta_{0}} e^{-\left(k^{2} \pi^{2}+\beta_{0}\right) t} \quad \sin k \pi z
\end{gathered}
$$

where $\bar{u}_{1}$ and $\bar{u}_{2}$ represent the step change from $\bar{u}_{1}$ to $\bar{u}_{2}$. The comparisons showed that a third-order lumped parameter model using the orthogonal collocation method gave an adequate dynamic system representation that can be used for subsequent controller design.

For a particular system in which $\beta_{0}=1.485$, the lumped system from the application of orthogonal collocation can be put in the state-space model form in which the matrices are as follows:

$$
A_{1}\left[\begin{array}{ccc}
-74.818 & 26.667 & -13.333 \\
16.667 & -22.818 & 16.667 \\
-13.333 & 26.667 & -74.818
\end{array}\right]
$$

Choosing the single temperature measurement location to be controlled at $z=0.5$, i.e. $y(0.5)=x_{2}$, which corresponds to one of the the collocation points, the output matrix $\mathbf{c}$ is then given by.

$$
c=\left[\begin{array}{lll}
0 & 1 & 0
\end{array}\right]
$$

Note that any other point of interest along the length of the heated rod could also be chosen. If this is not one of the collocation points, then the output matrix $C$ is determined by application of the Lagrange interpolation technique [35]. The corresponding transfer function of this system is given by:

$$
g(s)=\frac{-6 s^{2}+102.2057 s+28966.55}{s^{3}+172.4540 s^{2}+7945.441 s+69017.63}
$$

This transfer function relates the effect of the manipulated variable, $\mathrm{u}$ (steam pressure to the steamchest), to the controlled variable which is the temperature at point $z=0.5$. The control system is shown schematically in Figure 2.

$$
\text { Air at } \mathrm{T}_{0}{ }^{\circ} \mathrm{C}
$$

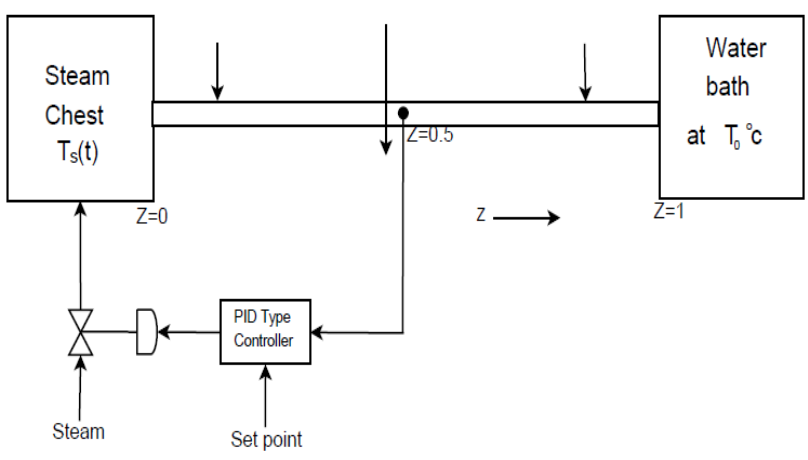

Figure 2: Schematic of metallic rod-temperature control system

\subsubsection{PID Controller Designs}

From the given transfer functions and the state space model, it is easy to check that the system is open-loop stable. This is a condition of the PID controller design method.

Solving for the quadratic roots of the numerator of the system transfer function, it is seen that the transfer function $g(s)$ has a RHP zero at $s=1 / \beta=78.5193$. Using the Type II factorization to factor out this RHP zero[20], it is straightforward to show that the fullorder, classic feedback controller equivalent to the IMC controller for $g(s)$ is given by

$$
g(s)=\frac{B_{n}}{B_{d}}
$$

where

$$
\begin{gathered}
B_{n}=s^{3}+172.4540 s^{2}+7945.441 s+69017.63 \\
B_{d}=471.1158 \varepsilon^{2} s^{3}+[28966.56] \varepsilon^{2} \\
+471.1158(2 \varepsilon+\beta) s^{2} \\
+28966.56(2 \varepsilon+\beta) s
\end{gathered}
$$

Putting this in the form of Eq. (17), one obtains that

$$
\begin{gathered}
b_{3}=1.0, \quad b_{2}=172.4540 \\
b_{1}=7945.441, \quad b_{0}=69017.63 \\
a_{3}=471.1158 \varepsilon^{2}, \\
a_{2}=28966.56 \varepsilon+471.1158(2 \varepsilon+\beta), \\
a_{1}=28966.56(2 \varepsilon+\beta)
\end{gathered}
$$

Using the appropriate equations in Section 4, the PI and PID-type controller parameter expressions follow directly from the relevant coefficient $a_{i}$ 's and $b_{i}$ 's given above. For the designs, $M^{*}=1.05$ was used.

\section{RESULTS AND DISCUSSIONS}

Closed-loop simulations of the PID-type controllers designed using the presented approach were carried out for unit step change in setpoint. In the earlier work[20], it has been shown that the proposed PID controller design method gives comparable performance with the full-order internal model controller, and much better than PID controllers designed based on model reduction to a first or secondorder system. Thus, this work being an exploratory application of an orthogonal collocation lumped-model of a distributed parameter system for PID controller design, performance comparison shall only be limited to the PID controllers designed using the classical Ziegler-Nichol tuning rules which is the starting point for industrial practitioners.

\subsection{Results}

\subsubsection{Nominal Closed-Loop Simulation Performance}

For a unit step change in setpoint, Figure 3 shows the plots of the simulated response of the PI controller 
designs, while Figure 4 shows a comparison of the PI controller designed by the method proposed in this paper, and that of a PI controller designed according to the Ziegler-Nichols tuning method.

For the same step change in setpoint as above, Figure 5 shows the plots of the simulated response of the ideal
PID controllers designed for the System, while Figure 6 shows a comparison of the performance of the ideal PID controllers (for $\varphi=0.6$ and 0.9 ) designed by the proposed method, and that of an ideal PID controller with Ziegler-Nichols tuning parameters.

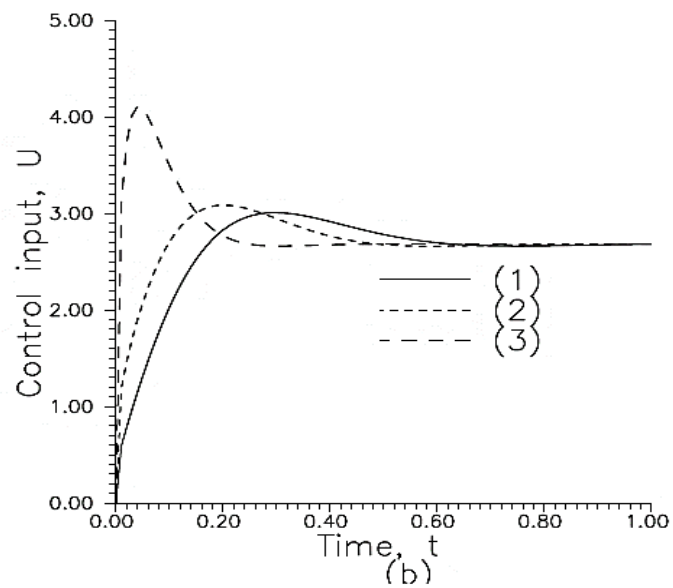

Figure 3: Response of the System to a unit step change in setpoint. Legend: 1,2,3 new PIdesign with $\varphi=$ 0.3, 0.6, 0.9, respectively. (a) Left: Controlled variable, (b) Right: Control input.
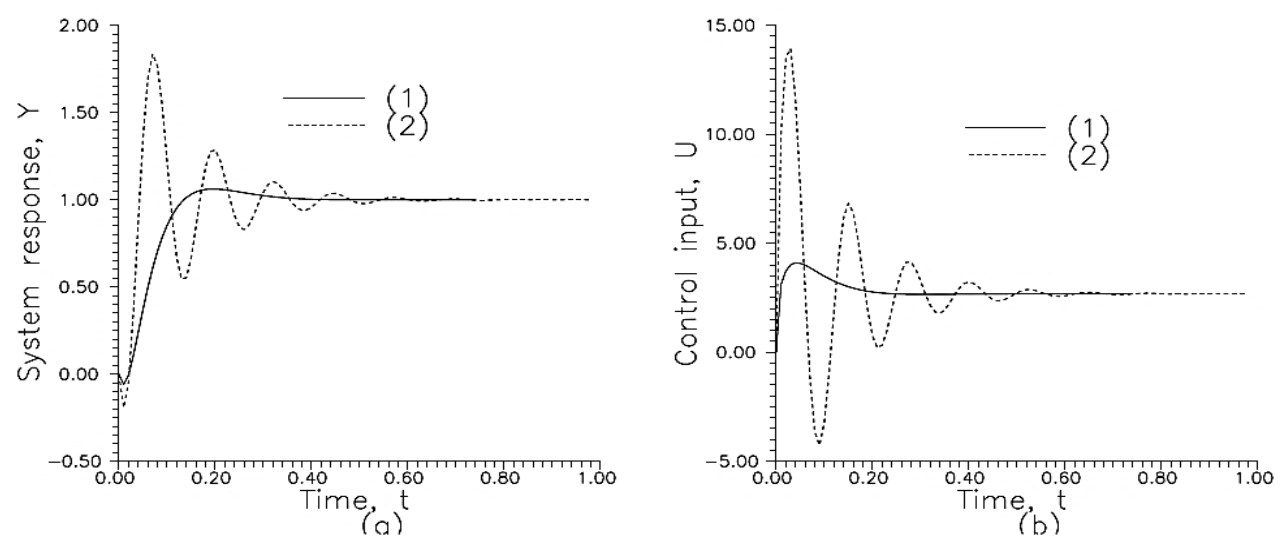

Figure 4: Comparison of performance of new PI controller with $\varphi=0.9$ (solid line) and ZN PI Controller (shortdashed line) for a unit step change in setpoint of the System. (a) Left: Controlled variable response, (b) Right: Control input response.
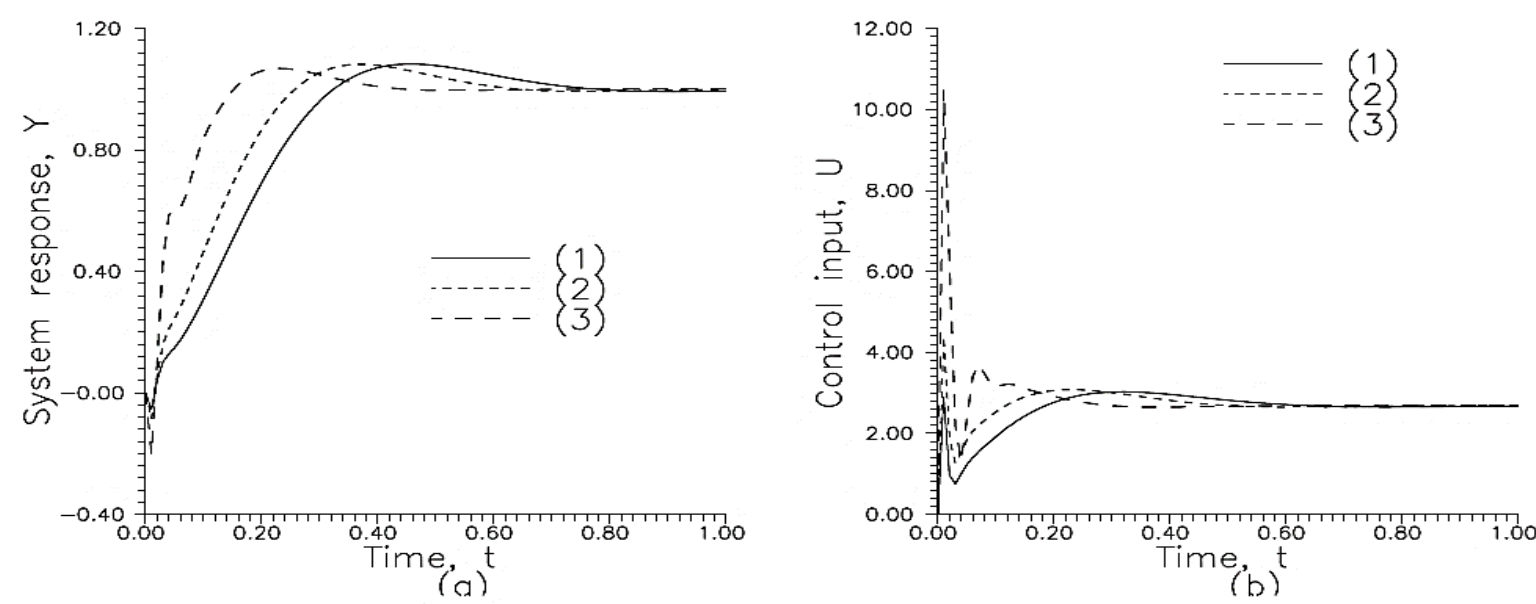

Figure 5: Response of System to a unit step change in setpoint. Legend: 1,2,3 proposed ideal PID design with $=0.3,0.6,0.9$, respectively. (a) Left: Controlled variable, (b) Right: Control input. 


\subsubsection{Effect of Plant/Model Mismatch}

The foregoing simulations were carried out on the 3rdorder model employed for controller design i.e. assuming a perfect model. In order to investigate the performance of the PID-type controllers designed by the method proposed in this paper to the effect of model/plant mismatch, they were all simulated on a 10th-order lumped model of the rod system (obtained by modal decomposition) which is assumed to be the plant. Figure 7 shows a comparison plot of the performance of the designed PI controllers (with $\varphi=$ $0.6,0.9$ ) on both the model used for controller design and the assumed 10th-order plant. The left figure shows the closed-loop system response, while the right figure shows the manipulated variable response. On the other hand, Figure 8 show the comparison of the performance of the closed-loop system response with the ideal PID controllers (designed by the proposed method), on both the 3rd-order model, and the assumed 10th-order plant for two different values of $\varphi$
: 0.9 (left figure), and 0.6 (right figure).

\subsection{Discussion}

\subsubsection{Nominal Performance}

The closed-loop simulation results shown in Figures 3 Figure 5 demonstrate the vastly superior performance of the PID controllers presented here, compared with those based on the classical Ziegler-Nichols tuning method. It can be seen that the proposed approach leads to PID controllers that drove the system output to the desired setpoint with smoother responses and shorter settling time. Additionally, it can be seen that the control inputs required by the PID-type controllers based on the Ziegler-Nichols tuning rules are highly oscillatory - implying high rate of control valve chatter. This is undesirable because it will result in faster rate of wear and tear on the valve seat. Conversely, control actions of the PID-type controllers designed by the proposed method are smooth and none oscillatory.
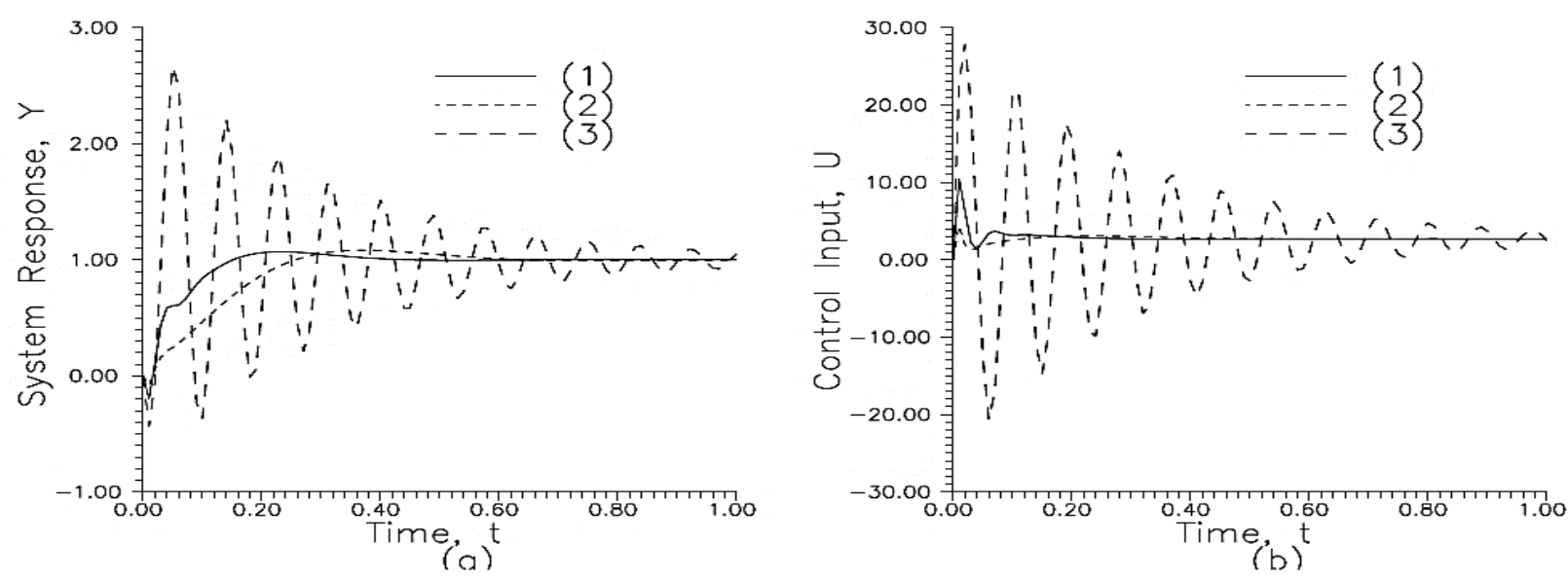

Figure 6: Comparison of performance of new ideal PID and ZN PID controllers. Legend: 1,2 new PID controller with $=0.9,0.6$, respectively; 3 ZN PID controller. (a) Left: Controlled variable response, (b) Right: Control input

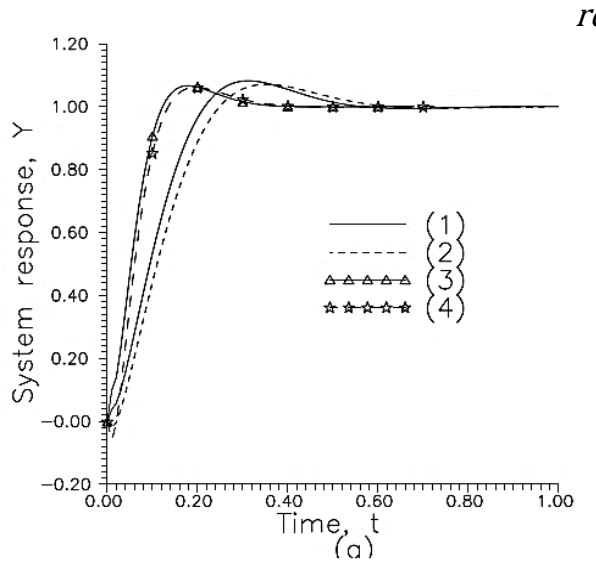
response.

Figure 7: Comparison plot of response of new PI controller on both model and plant to a unit step change in setpoint of the System. Legend: 1, plant and 2, model for $\varphi=0.6$; 3, plant and 4, model for $\varphi=0.9$. (a) Left:

Controlled variable, (b) Right: Control input. 

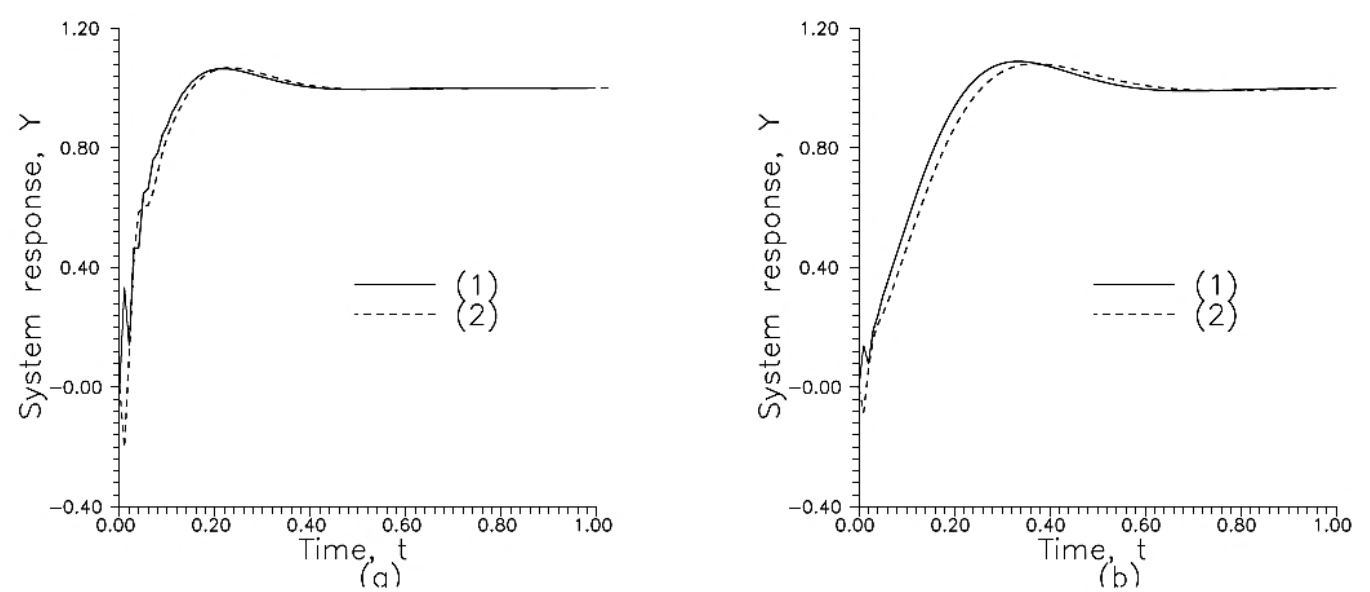

Figure 8: Comparison plot of performance of new ideal PID controller on both model and plant to a unit step change in setpoint of the System. (a) Top legend: 1, plant and 2, model for controller at $\varphi=0.9$; (b) Bottom legend: 1, plant and 2 , model for controller at $\varphi=0.6$.

It can also be seen that the value of the parameter $(\varphi)$ influences the closed-loop dynamic response of the system. As the $\varphi$ is increased, the closed-loop response of the system becomes faster, and the corresponding control input also increases. Although not considered as part of the scope of present work, it is conceivable that one can setup an optimization problem to choose an optimal $\varphi$ that minimizes a desired objective function which defines the closed performance such as Integral Squared Error (ISE) or Integral Absolute Error (IAE)

\subsubsection{Effect of Plant/Model Mismatch}

The closed-loop simulation results shown in Figure 7 and Figure 8 indicate that the designed controllers are not sensitive to the plant/model mismatch considered. The controllers designed by the proposed method still drove the output response to the desired points in a satisfactory manner.This is an attractive feature since there is always a mismatch between models employed for controller design and the actual plant where this would be implemented. Thus, it is not desired that the controllers be highly sensitive to modelling errors as this could lead to significant deterioration of closedloop performance or the closed-loop system could even become unstable. Although the closed-loop simulation results are presented at only two parameter values of $\varphi$, closed-loop simulation results at other values showed the same trend.

\section{CONCLUSION}

This paper has shown that using the orthogonal collocation technique is an attractive method to convert the parabolic partial differential equation modelling the one-dimensional heated rod into a low- order lumped parameter model, which was then directly employed for controller design based on a method previously developed by the authors. Closed loop simulation results showed the superior performance of the proposed PID controllers compared with the PID controllers based on the classical ZieglerNichols method. The approach described for the distributed one-dimensional heated rod system presented in the paper is quite general and may be used to carry out PID-type controller designs for other single-input, single-output distributed parameter systems. Consequently, the entire procedure is being developed into a computer-aided design environment to make it widely attractive for adoption by industrial practitioners and other researchers that may be working in this area. The computer aided design package to do this shall be subject of a future paper.

\section{REFERENCES}

[1] Ray, W. H. Advanced Process Control, McGraw-Hill, 1981.

[2] Lienhard, J. H. IV and Lienhard, J. H. V. A Heat Transfer Textbook, 3rd ed. Phlogiston Press, 2000.

[3] Wendl, M. C. The Theoretical Foundations of Conduction and Convection Heat Transfer, The Wendl Foundation, 2012.

[4] Martinez, I. "Heat Conduction.", http://webserver. dmt.upm.es/ isidoro/, Accessed on Aug. 20, 2018.

[5] Zhou,W., Hamroun, B., Gorrec, Y. L., and Couenne, F. "Dissipative boundary control systems with application to an isothermal tubular reactor," IFACPapersOnLine, vol. 28, no. 13, jan, pp. 150-153, 2015.

[6] Williams, A. O. F. and Adeniyi, V. O. "Modeling and Simulation of the Dynamics of Steam-heated Heat Exchangers Using Orthogonal Collocation," Modelling, Simulation \& Control, vol. 35, no. 1, pp. 
45-64, 1991.

[7] Finlayson, B. A.The Method of Weighted Residuals and Variational Principles - With Application in Fluid Mechanics, Heat and Mass Transfer, Academic Press, 1972.

[8] Simon, L. and Loney, N. W. "Mathematical Modeling and Process Control of Distributed Parameter Systems: Case Study. The One-Dimensional Heated Rod," Chemical Engineering Education, vol. 37, no. 2, pp. 126-131, 2003,

[9] Curtain, R. and Morris, K. "Transfer functions of distributed parameter systems: A tutorial," Automatica, vol. 45, no. 5, may, pp. 1101-1116 2009.

[10] Li, H. X. and Qi, C. "Modeling of distributed parameter systems for applications - A synthesized review from time-space separation," Journal of Process Control, vol. 20, no. 8, pp. 891-901, 2010,

[11] Marsili-Libelli, S. "Optimal design of PID regulators," International Journal of Control, vol. 33, no. 4, pp. 601-616, apr 1981.

[12] Brambilla, A., Scali, C., and Chen, S. "Tuning of Conventional Controllers for Robust Performance," IFAC Proceedings Volumes, vol. 22, no. 18, nov, pp. 123-128, 1989.

[13] Shinskey, F. G., "Reflections on CPC-III," in Chemical Process Control-CPC III, Eds., M. Morari, and T. J. McAvoy, Elsevier, 1986

[14] Ziegler, J. G. and Nichols, . B. "Optimum Settings for Automatic Controllers," Trans. ASME, vol. 64, no. 11, , pp. 759 - 768, 1942.

[15] Cohen, G. H. and Coon, G. A. "Theoretical Considerations of Retarded Control," ASME Trans, vol. 75, pp. 827-836, , 1953.

[16] Luyben,W. L. Process Modeling, Simulation, and Control for chemical Engineers, 2nd ed., McGrawHill., 1990.

[17] Rivera, D. E., . Morari, M., and Skogestad,S. "Internal Model Control: PID Controller Design,” Industrial \& Engineering Chemistry Process Design and Development, vol. 25, no. 1, jan, pp. 252-265, 1986.

[18] Morari, M. and Zafiriou, E. Robust Process Control. Prentice-Hall, 1989.

[19] Ogunnaike, B. A. and Ray, W. H. Process Dynamics, Modelling, and Control, Oxford Univ. Press, 1994.

[20] Williams, A. O. F. and Adeniyi,V. O. "A New Method for the Design of PID-Type Controllers," IFAC Proceedings Volumes, vol. 29, no. 1, pp. 6125-6130, 1996,.

[21] Chen, C. L. "Tuning of PI Controllers for SelfRegulating Processes," J. Chin. Inst. Chem. Engrs., vol. 28, pp. 313-327, 1997.

[22] Chen, C. L., Huang, H. P. and Hsieh, C. T. "Tuning of PI/PID controllers based on specification on maximum closed-loop amplitude ratio," Journal of Chemical Engineering of Japan, vol. 32, no. 6, pp.
783-788, 1999.

[23] Chen, D and Seborg, D. E. “PI/PID Controller Design Based on Direct Synthesis and Disturbance Rejection," Industrial \& Engineering Chemistry Research, vol. 41, no. 19, sep, pp. 4807-4822, 2002.

[24] Skogestad, S. "Simple Analytic Rules for Model reduction and PID controller tuning," Journal of Process Control, vol. 13, no. 4, pp, pp. 291-309. 2003,.

[25] Vilanova, R. "IMC based Robust PID design: Tuning guidelines and automatic tuning," Journal of process Control, vol. 18, no. 1, pp. 61-70, 2008.

[26] Skogestad, S. and Postlethwaite, I. Multivariable Feedback Control Analysis and Design. John Wiley, 2005.

[27] Seborg, D. E., Mellichamp, D. A., Edgar, T. F. and Doyle III, F. J. Process Dynamics and Control, John Wiley \& Sons, 2010.

[28] Mikhalevich, S. S., Baydali, S. A. and Manenti, F. "Development of a tunable method for PID controllers to achieve the desired phase margin," Journal of Process Control, vol. 25, jan, pp. 28-34, 2015.

[29] Umamaheshwari, G., Nivedha, M and Dorritt, J. P., "Design of Tunable Method for PID Controller for Higher Order System," International Journal of Engineering And Computer Science, vol. 5, no. 7, pp. 17239-17242, 2016.

[30] Visioli, A. "Research trends for PID controllers," Acta Polytechnica, vol. 52, no. 5, pp. 144-150, 2012.

[31] Yamaguchi, N. and Kanoh,H. "Design of Robust PID Parameters for Distributed Parameter Processes," IFAC Proceedings Volumes, vol. 33, no. 24, sep, pp. 221-226, 2000.

[32] Hulkó, G. and Belavy, C. "PID control of Dstributed Parameter Systems," IFAC Proceedings Volumes, vol. 36, no. 18, pp. 101-106, 2003.

[33] Alvarez, J. D., Normey-Rico, J. E. and Berenguel, M. "Design of PID Controller with Filter for Distributed Parameter Systems," in IFAC Proceedings Volumes (IFAC-PapersOnline), vol. 2, no. PART 1. Elsevier, jan, pp. 495-500, 2012.

[34] Mikhalevich, S. S., Rossi, F., Manenti, F., Baydali, S. A. "Robust PI/PID Controller Design for the Reliable Control of Plug Flow Reactor," Chemical Eng. Transactions, vol. 43, pp. 1525-1530, 2015.

[35] Villadsen, J. and Michelsen, M. L. Solution of Differential Equation Models by Polynomial Approximation, Prentice-Hall, 1978.

[36] Garcia, C. E. and Morari, M. "Internal Model Control. A Unifying Review and some New Results," Industrial \& Engineering Chemistry Process Design and Development, vol. 21, no. 2, pp. 308-323, 1982,.

[37] Forstythe, G. Malcom, M. and Moler, C. Computer methods for Mathematical Computations," Prentice-Hall, 1977. 